\title{
Research on Ideological Change of Management of College Teachers based on Analytic Hierarchy Process
}

\author{
Liu Liyun ${ }^{1, a}$, Feng Guofei ${ }^{2}$, Wang Yan ${ }^{1, b}$ \\ ${ }^{1}$ Shijiazhuang Vocational Technology Institute Economic and Trade Department ,Shi jiazhuang \\ 050081, China \\ ${ }^{2}$ Ordnance Engineering College, Shi jiazhuang,050003,China \\ a, liuliyun2013@yeah.net, ${ }^{b, 461964990 @ q q . c o m ~}$
}

Keywords: College teacher, Management, Analytic hierarchy process

\begin{abstract}
Management of teachers of colleges and universities is very important in education. Traditional teacher management focuses on the quality the teachers, and the purpose of education is training good students. However, teacher is the key part in education, and we should take the culture of teachers themselves into consideration. In this paper, we propose to change the ideology of teacher management from training students to consider both students and teachers. We analyze the management of teachers based on analytic analysis process, and also analyze our method with Empirical Analysis.
\end{abstract}

\section{Introduction}

Teachers are not only the main body of the development of colleges and universities, but also the most important resources and source of competitive. The concept of the "people-oriented" management is a necessary requirement for teachers in colleges and universities, which will help to make a comprehensive progress. However, traditional college teacher management isn't people-oriented, and college teachers are treated as resources, and thus teacher management is considered to be configuration of resources, which greatly reduces the teacher's teaching initiative [1]. Hence, a good management of college teachers should not only consider the quality of teaching, but also take the culture of the teacher itself into consideration. Hence, people should change their ideology of college teachers from traditional resource configuration to the concept of teacher-oriented.

In multi-objective decision making, there will be some systems of many variables, the complex structure and the role of uncertainties significant characteristics [2]. The problem of decision-making in these complex systems is necessary to make a correct valuation of the relative degree of importance to describe the target. However, the degree of importance of each factor is not the same. In order to reflect the relative importance of the factors, people need to evaluate the importance of each factor (i.e. weights), and the collection of weights of each factor weight is called the weight set. Weight is an objective reflection of the physical attributes of the indicators, and is also the result of subjective and objective comprehensive measure.

Analytic Hierarchy Process (AHP) is a better method for ensuring the weights. It divides the factors into a relative ordered hierarchy, more principled multi-object and multi-rules decision making method, and is also a method of the composition of quantitative and qualitative analysis. In this paper, we believe that the ideology of college teacher must change from the concept configuration to the concept of teacher-oriented, and use the analytic hierarchy process to analyze the change of this ideology.

The rest of the paper is organized as follows. In section 2, we review related work about the management of college teachers and the applications based on the analytic hierarchy process. Theory of analytic hierarchy process is given in section 3. Empirical Analysis and conclusion are given in section 4 and 5, respectively. 


\section{Related work}

In this section, we review related works about the management of college teachers and the applications based on the analytic hierarchy process.

$\mathrm{Li}$ and $\mathrm{Li}$ [3] investigate the evaluation problems for Chinese college teacher performance appraisal system in which the attribute weights take the form of real numbers, and the preference values take the form of uncertain information. By utilizing some operational laws of uncertain variables, they extended the TOPSIS method to evaluation problems for Chinese college teacher performance appraisal system in uncertain setting. Finally, an illustrative example demonstrates the practicality and effectiveness of the proposed method.

The bifactor structure has been considered a credible model for various types of data. Wang and Gao [4] propose to test the utility of the bifactor IRT model for dimensionality assessment, and to confirmatory factor analyze the structure of the newly-developed scale, the Job Burnout Scale for Chinese College Teachers. 1331 teachers were chosen from 11 various Universities in China. 1111(83.47\%) were valid. Compared to the unidimensional model, the bifactor model resulted in a statistically improved model fit. The Full-Information Item Bifactor solution provides an excellent alternative.

Li and Yuan [5] simplify the AHP algorithm by the method of approximation algorithms through the study of the theory and algorithms of analytical hierarchy process. Meanwhile, AHP is introduced to the study of sports events risk. A multi-level risk index system is established, and relative importance of risk factors is determined. More objective judgment of the risk situation of risk factors in the events is made and events risks are systematically analyzed from the perspective of evaluation combined with the risk characteristics of sports events. Then the quantitative relationship among the comparison of the projects is given from the perspective of mathematical analysis, with the analysis of the results given by the expert survey method, through specific examples.

The academic circle and government sector has attached importance to the statistics and evaluation of sports industry. Hence, to design an index system, which matches with the operational mechanism of sports industry and has a wide coverage and reasonable structure, and to establish a comprehensive evaluation index system on the development level of sports industry has important realistic and theoretical significance. Hence, Huang and Zhi [6] collect documents and uses AHP to establish and evaluate the index system for the development level of principal sports industry.

\section{Theory of analytic hierarchy process (AHP)}

AHP is a combination of quantitative and qualitative system analysis method proposed by the professor T. L. Satty at the University of Pittsburgh in the early 1750s. AHP divides various factors of the complex issues into interrelated orderly hierarchy, make it more principled. Based on the judgment of certain objective reality, it presents quantitatively the relative importance of each factor in each level, and uses the mathematical methods to determine the weights of factors in each level in order to provide a scientific basis for the correct evaluation of the research project.

\section{a. Build a Hierarchical evaluation system}

By analyzing the relationship of each index, build a hierarchical evaluation index system, as shown in table 1.

Table 1. The second order evaluation index system

\begin{tabular}{cccc}
\hline $\mathrm{u} 1$ & $\mathrm{u} 2$ & $\ldots$ & $\mathrm{um}$ \\
\hline $\mathrm{u} 12$ & $\mathrm{u} 21$ & $\ldots$ & $\mathrm{um} 1$ \\
$\mathrm{u} 13$ & $\mathrm{u} 22$ & $\ldots$ & $\mathrm{um} 2$ \\
$\ldots$ & $\ldots$ & $\ldots$ & $\ldots$ \\
$\mathrm{u} 1 \mathrm{n}$ & $\mathrm{u} 2 \mathrm{n}$ & $\ldots$ & $\mathrm{umn}$ \\
\hline
\end{tabular}

\section{b. Build a pairwise comparison judgment matrix}

After the evaluation index system is established, the affiliation between the upper and lower-level indicators is identified. On the same level indicators, compare every index with the others, and 
present the comparison results with the one scaling method. The meaning of the scale in all levels is shown in table 2.

Table 2. Meaning of 1-9 mark degree

\begin{tabular}{cc}
\hline Scale & Meaning \\
\hline 1 & Two factors have the same importance. \\
3 & One factor is a little more important that another factor. \\
5 & One factor is obviously more important that another factor. \\
7 & One factor is strongly more important that another factor. \\
9 & One factor is extremely more important that another factor. \\
$2,4,6,8$ & Value of the judgment for the above adjacent. \\
\hline
\end{tabular}

Then, we can obtain a pairwise comparison judgment matrix $A=\left\{a_{i j}\right\}$ for $n$ indexes in one same level, and the values in the judgment matrix should satisfy the following condition:

$$
a_{i j}>0, a_{i j}=\frac{1}{a_{i j}}, a_{i j}=1
$$

\section{c. Compute index weight}

(1) Calculate the plot of each row of the judgment matrix $A, M_{i}: M_{i}=\prod_{j=1}^{n} a_{i j}, i=1,2,3, \cdots, n$.

(2) Calculate the value of the n-th root of $M_{i}$ in each row, $\bar{W}_{i}=\sqrt[n]{M_{i}}, i=1,2,3, \ldots n$, where $n$ is the of order the matrix.

(3) Normalize the vector $\left[\begin{array}{lllll}W_{1} & W_{2} & \cdots & W_{n}\end{array}\right]^{T}$ with the formula $W_{i=1}=\frac{W_{i}^{n} \bar{W}_{i}}{W^{n}}$, where $W_{i}$ is the weight of the calculated index.

d. Compute maximum eigenvalue $\lambda$ max of the judgment matrix $A$

$$
\lambda_{\max }=\sum_{i=1}^{n} \frac{(A \cdot W)_{i}}{n W_{i}}
$$

$A \cdot W=\left[\begin{array}{cccc}a_{11} & a_{12} & \cdots & a_{1 n} \\ a_{21} & a_{22} & \cdots & a_{2 n} \\ \cdots & \cdots & \cdots & \cdots \\ a_{n 1} & a_{n 2} & \cdots & a_{n n}\end{array}\right] \cdot\left[\begin{array}{c}W_{1} \\ W_{2} \\ \cdots \\ W_{n}\end{array}\right]$, and $(A \cdot W)_{i}=a_{i 1} W_{1}+a_{i 2} W_{2}+\cdots+a_{i n} W_{n}$.

\section{e. Consistency test}

(1) Calculate the consistency index $C_{l}, C_{l}=\lambda_{\max }-\frac{n}{n-1}$.

(2) Check the average consistency index of the matrix of the same order $R_{l}$ (in table 3).

Table 3. Average random coherence index

\begin{tabular}{cccccccccc}
\hline Order number n & 1 & 2 & 3 & 4 & 5 & 6 & 7 & 8 & 9 \\
\hline$R_{\iota}$ & 0 & 0 & 0.58 & 0.90 & 1.12 & 1.24 & 1.32 & 1.41 & 1.45 \\
\hline
\end{tabular}

(3) Calculate the consistency rate $C_{R}$.

$C_{R}=\frac{C_{l}}{R_{l}}$.

When $C_{R} \geq 0.1$, A has non- satisfactory consistency, and should be adjusted or abandoned. According to assign and adjust all indexes, the tendentious and flexibility of the evaluation index system of the evaluation user can be presented.

\section{Empirical Analysis}

This topic of teacher management quality evaluation includes peer teacher evaluation, student evaluation, the expert evaluation and evaluation of teachers themselves. In the following, we will 
separately establish its hierarchical relationships and evaluation index of teacher management quality evaluation.

\section{Result}

The aim of this research is to analyze the risk affecting factors of the teacher management, and the literature research and survey questionnaire are carried out to have the risk evaluation. The corresponding counter measurements can be established, and the quality of the green marketing can be improved. The 45 expert questionnaires were extended, and the 40 questionnaires were recalled, and the recall rate is $89 \%$.

\section{Conclusion}

Management of teacher of colleges and universities is very important in our society. Teacher-oriented management concept should be considered in this field. In teacher management, peer evaluation, student evaluation, expert evaluation and teachers' self-evaluation are four main parts that decide the quality of management. In this paper, we propose to change the ideology of teacher management from training students to consider both students and teachers. We analyze the management of teachers based on analytic analysis process, and also analyze our method with Empirical Analysis.

\section{References}

[1] Tuo Yunting, "The Study on Human Resource Management Performance Evaluation based on Data Envelopment Analysis and Analytic Hierarchy Process", JDCTA: International Journal of Digital Content Technology and its Applications, Vol. 6, No. 14, pp. 256 265, 2012.

[2] Zongxiang Liu, "A Comprehensive Evaluation Method for Physical Education Teaching Based on Analytic Hierarchy Process", JDCTA: International Journal of Digital Content Technology and its Applications, Vol. 7, No. 2, pp. 404 411, 2013.

[3] Xiaofei Li, Dong Li, "TOPSIS Method for Chinese College Teacher Performance Appraisal System with Uncertain Information", AISS: Advances in Information Sciences and Service Sciences, Vol. 3, No. 6, pp. $59 \sim$ 64, 2011.

[4] Peng Wang, Fengqiang Gao, "Full-Information Item Bifactor Analysis of the Job Burnout Scale for Chinese College Teachers", JCIT: Journal of Convergence Information Technology, Vol. 5, No. 7, pp. $155 \sim$ 160, 2010.

[5] Li Yue-hui, Yuan Yan, "Research on Risk Management of Sports Events Based on Analytic Hierarchy Process", JDCTA: International Journal of Digital Content Technology and its Applications, Vol. 7, No. 2, pp. 124 132, 2013.

[6] Huang Zhonghui, Zhi Chuan, "A Comprehensive Evaluation Method for Sports Modernization based on Analytic Hierarchy Process", JDCTA: International Journal of Digital Content Technology and its Applications, Vol. 7, No. 3, pp. 80 86, 2013. 